diagnose a second case of this nature I doubt, but I think the history of it gives some points of diagnostic value.

Loughborough.

\section{A CASE OF AUTO-VACCINATION.}

By George H. Ormsby, M.R.C.P. IreL., \&O.

THE accompanying illustration shows a novel effect of vaccination on a patient at present under my care.

A married man, aged 45 years, while at work on Sept. 14th, 1900, got a slight scratch on the outer angle of the right eye. Abjut a week previously I had vaccinated his child. His wife being ill he was obliged to take the child at night, the result being that matter from the arm inoculated the scratch. I first saw him on Sept 24th and the following day had a photograph taken. His eye was completely closed

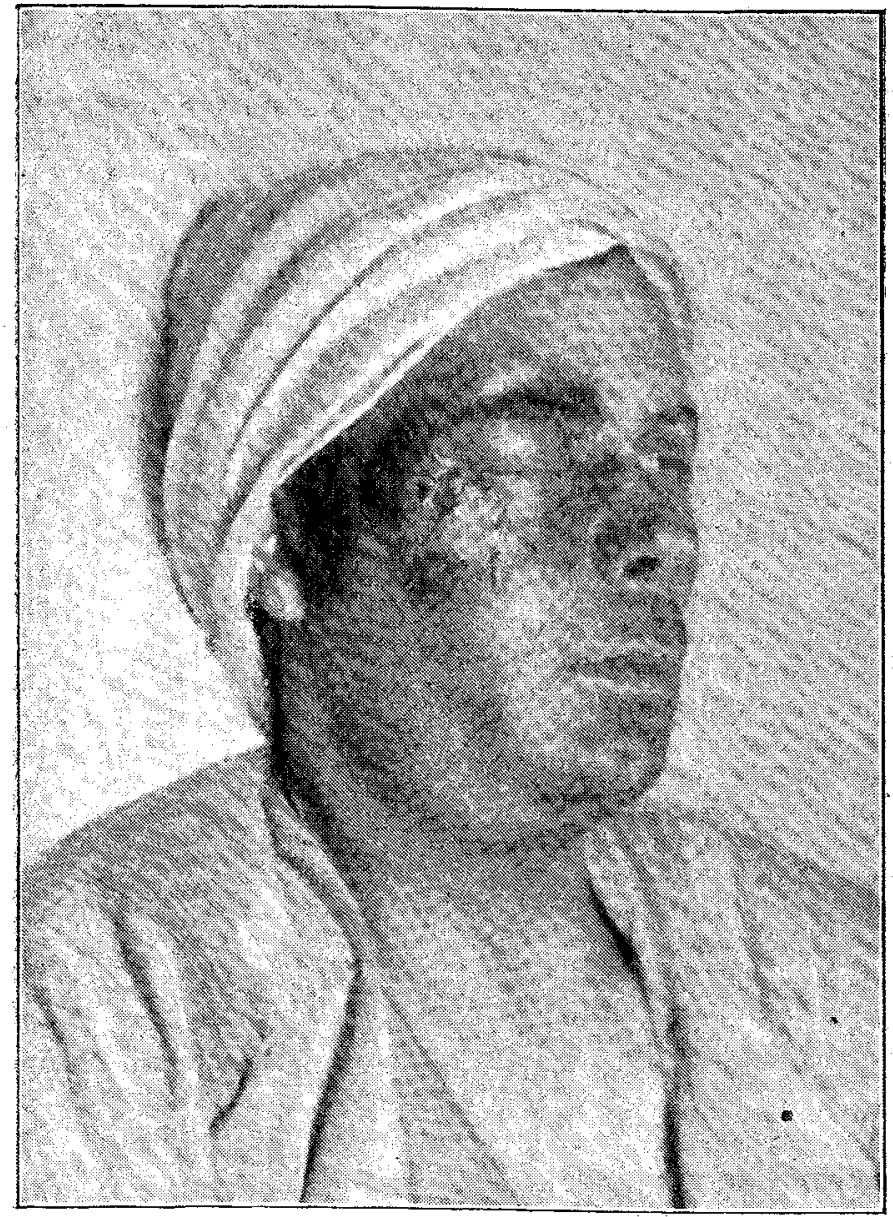

and was very painful and irritable. His symptoms the same night were so suggestive of septic poisoning that I asked Mr. F. MacD ongall to see him with me. By the following morning, however, he was much better and continued to improve steadily. The dark ring round the eye is owing to its being painted with iodine.

Mr. MacDougall tells me that during his extensive experience as "public vaccinator" he has never seen a similar case.

Runcorn.

\section{NASAL DIPHTHERIA MASKED BY MEASLES.}

By WilliaM WrLlys, M.R.O.S. ENG., L.R.C.P. LOND, L.S.A.

I HAVE had under my care a patient whose illness in some respects resembled the case of diphtheritic paralysis reported by Dr. A. J. Rice Oxley ${ }^{1}$ where only a slight sore-throat had been complained of and some difficulty had been experienced in diagnosing the condition of the patient. My patient, a boy aged five years, had been suffering from a typical attack of morbilli, his little sisters, aged three and two years respectively, being at the same time similarly affected.

3 The Laycet, Feb. 2nd, 1901, p. 321.
The only suspicious circumstance was the protracted and obstinate nature of the nasal catarrh, which was purulent, and in the case of the elder girl bad existed for two weeks prior to the appearance of the measles rash. In no instance was sore-throat complained of nor was any membrane found in the fauces. The two girls made a perfect and permanent recovery, but the boy about a week atter the subsidence of all symptoms and sjgns of measles became so weak and debilitated that I ordered him back to bed. In a few days. however, he appeared better and was allowed to get up, and as the weather was fine he was able to go out of doors about a week later, when he walked to my house though still obviously weak. Ten days afterwards his father carried him to me, as he said that the child seemed to have lost the nse of his legs, toppling abont when trying to walk, and that his head drooped to the left side. I accordingly once more ordered him to bed, and on my visit next day 1 found that he had a cough of a clashing, hollow character, which was quite ineffective in bringing up any phlegm, though after repeated paroxysms he retched and expelled mucus and food through his nose. My suspicions being aroused I examined his palate and found it to be paralysed as were also the muscles of extraordinary expiration. The patellar tendon reflexes could not be elicited and his pupils were dilated and sluggish to light. His expression was vacant and there was left internal strabismus, Bronchitis supervened, and though of itself not of a serious character it was rendered so by the impaired expiratory power. The boy's condition soon became critical, the repeated paroxysms of cough, coupled with the sitting posture occasioned by orthopnca, telling severely upon the circulatory system. The treatment consisted of gradually increasing doses of liquor strychnix and liquor ferri perchloridi three times a day and friction of the chest muscles with camphorated oil. Under it the child's condition improved so much that in three weeks he could sit up comfortably in bed and play with his toys. Two further attacks of catarrhal bronchitis occurred and, though obstinate, were satisfactorily dealt with by stimulant expectorants and the use of the steam-kettle. The boy's hearing was markedly impaired on both sides during convalescence, but gradually improved, as did his power of deglutition, and the "twangy "nature of his voice diminished. As paresis of the chest muscles persisted and the legs were much wasted, and the knee-jerks were still absent at the end of six weeks, massage for 30 minutes followed by galvanism was employed once a day for a fortnight and a rapid improvement was soon manifest, the boy's respiration becoming more natural, and, the use of his legs returning, he was able to walk a little, though in a tottering way. A year has elapsed, and he now enjoys very fair health except for occasional bronchitic attacks. He walks and runs well, his hearing is normal, he speaks with but a slight nasal twang, the thoracic movements are good, the knee-jerks have returned though only in a minor degree, and the strabismus is only obvious when he is over-tired.

The chief interest in this case centres in the fact that the patient undoubtedly bad nasal diphtheria coincidently with measles, and that although his sister had a far worse purulent nasal discharge both during and before her attack of measles she developed no post-diphtheritic paralysis. The rarity of the concurrence of diphtheria with measles makes these notes worth publishing.

Great Yarmouth.

Infectious Diseases Notification Act.-At Falmouth on Feb. 11th a man was fined £1 $3 s$. $6 d$. for not notifying that bis child had scarlet fever. The medical officer of health stated that three other cases of fever were traced to this one. The defence was that a medical man was not required for such a trifling case.

Plymodth Milk.supply. - At the meeting of the Plymouth Borough Conncil held on Feb. 11th it was decided that the medical officer of health be authorised to issue annual certificates to dairy farmers residing either within or withont the borough who supplied milk, cream, and butter to the inhabitants of Plymouth and who were willing to have their farms inspected. A list of dairy farmers to whom certificates shall have been granted will be published for the information of the residents of Plymouth every three months. This seems a wise decision of the council, for the recent typhoid fever outbreak at Plymouth was traced to contaminated milk. 88 cases occurred, 11 of which terminated fatally. 